Case Report

\title{
Cavernous Malformations of Septum Pellucidum and Trigemi- nal Nerve: A Case Report
}

\author{
Yugandhar Samireddypalle ${ }^{1}$, Ravikumar Hanumaiah ${ }^{1,2}$ \\ ${ }^{1}$ Columbia Asia Hospital, Whitefield, Bangalore, Karnataka, India \\ 2 SUNY Upstate Medical University, Syracuse, New York, USA \\ * Correspondence: h.ravi.k@gmail.com
}

How to cite this paper: Samireddypalle, Y., \& Hanumaiah, R. (2021). Cavernous Malformations of Septum Pellucidum and Trigeminal Nerve: A Case Report. Global Journal of Medical Case Reports, 1(1), 1-4. DOI:10.31586/gjmcr.2021.010101 Retrieved from

https://www.scipublications.com/journal/in-

dex.php/gjmcr/article/view/29

Received: May 19, 2021

Accepted: June 22, 2021

Published:June 23, 202

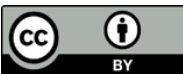

Copyright: (c) 2021 by the authors. Submitted for possible open access publication under the terms and conditions of the Creative Commons Attribution (CC BY) license (http://creativecommons.org/li-

censes/by/4.0/).

\begin{abstract}
Cerebral cavernous malformations are commonly seen in the cerebral parenchyma and are extremely rare in septum pellucidum, with only few case reports in the literature. Similarly cavernous malformation involving cranial nerves is also rare. We report a case of cavernous malformations involving both septum pellucidum and trigeminal nerve in a 73 years old gentleman, diagnosed with dementia two years back, and presented with history of recurrent falls.
\end{abstract}

Keywords: Cavernous malformations, Septum pellucidum, Trigeminal nerve, Superficial siderosis, Hydrocephalus

\section{Introduction}

Cerebral cavernous malformations are hamartomatous venous malformations of the brain. They are commonly identified in the cerebral parenchyma and are frequently multiple. They occur rarely in the ventricular system and are even rarer in septum pellucidum. Similarly cavernous malformation along the cranial nerves is also rare with only few case reports in the literature. We report a case of cavernous malformations involving both septum pellucidum and trigeminal nerve.

\section{Case Report}

A 73 years old gentleman, known case of hypertension and diabetes mellitus on regular medications, came to the hospital with history of recurrent falls since 2 months. He had been diagnosed with dementia two years back, which has been gradually progressing. Clinical examination revealed antalgic gait. No focal neurological deficit.

Non contrast CT brain demonstrated ventriculomegaly, disproportionate to the sulcal prominence probably representing changes of normal pressure hydrocephalus. A 1.9 $x 1 \mathrm{~cm}(\mathrm{AP} x \mathrm{TR})$ oblong hyperdense lesion was seen along the septum pellucidum (Figure $1 \mathrm{a}, \mathrm{b}$ ) with a few specks of coarse calcification. Another triangular hyperdense lesion was identified along the right trigeminal nerve (Figure $1 \mathrm{c}, \mathrm{d}$ ).

MR brain with contrast was performed for further characterization of these lesions. MRI revealed ventriculomegaly with flow void in the region of aqueduct, suggesting Normal pressure hydrocephalus.

The septum pellucidum lesion was T1 isointense and predominantly T2 hypointense with central popcorn appearance and marked blooming on SWI images. Mild enhancement of the lesion was identified on post contrast images (Figure 2).

The right trigeminal nerve lesion was not well identified on the conventional T1, T2 and T1 post contrast sequences, but was well appreciated on SWI sequence, which revealed linear / oblong hyperintensity on the SWI phase images (Figure 3).

SWI sequences demonstrated hypointensities along the cerebellar foliae, representing superficial siderosis, indicating that the trigeminal nerve cavernous malformation had 
bled previously. Multiple Tiny SWI hypointensities were seen distributed in bilateral cerebral hemispheres, suggesting microbleeds (Figure 4).
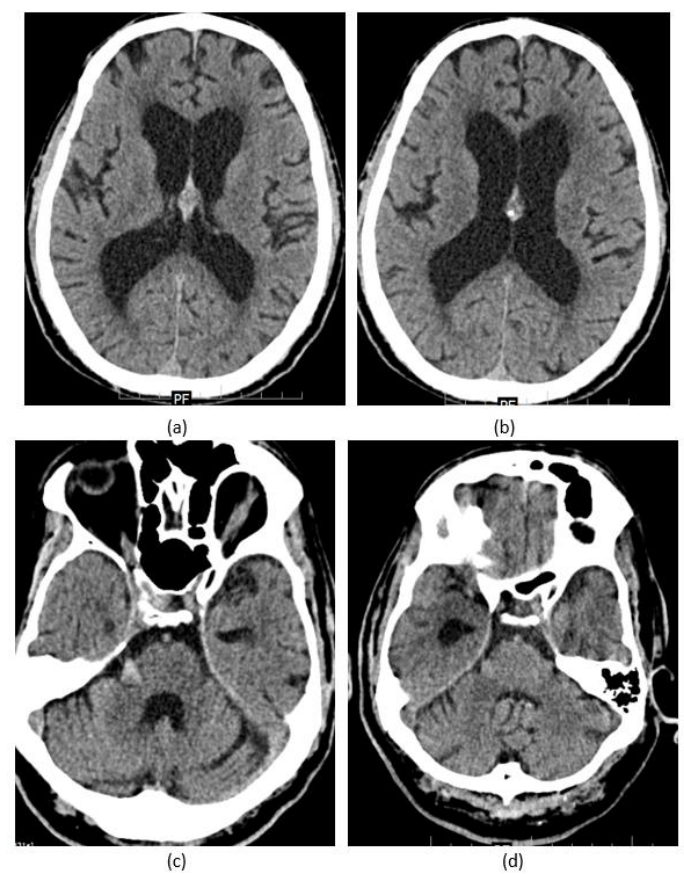

Figure 1. Non contrast CT brain demonstrates a $1.9 \times 1 \mathrm{~cm}(\mathrm{AP} \times \mathrm{TR})$ oblong hyperdense lesion along the septum pellucidum (Figure 1a, b) with a few specks of coarse calcification. Another triangular hyperdense lesion was identified along the right trigeminal nerve (Figure $1 \mathrm{c}, \mathrm{d}$ ). There is ventriculomegaly, disproportionate to the sulcal prominence probably representing changes of normal pressure hydrocephalus.

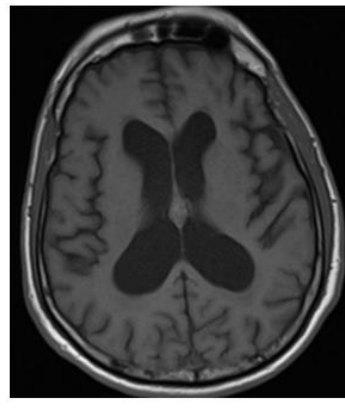

(a)

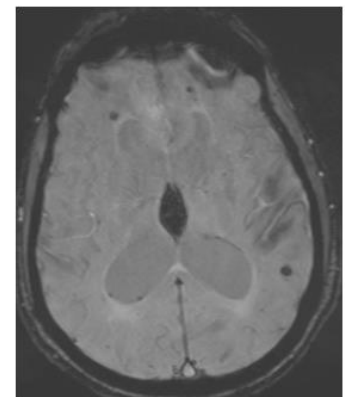

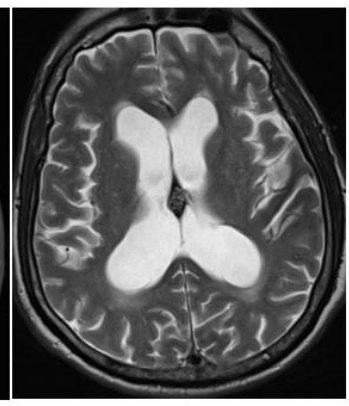

(b)

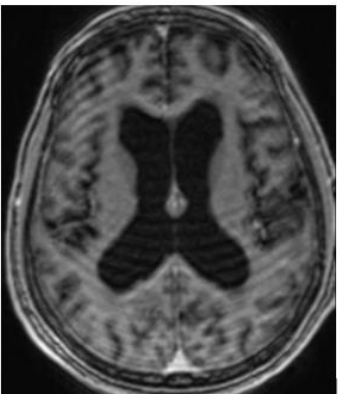

Figure 2. MRI images demonstrating T1 isointense lesion in the septum pellucidum (a) which is predominantly hypointense on T2 WI (b) with central popcorn appearance and marked blooming on SWI images (c) suggestive of cavernous malformation. Mild enhancement of the lesion was identified on post contrast images $(\mathrm{d})$. 


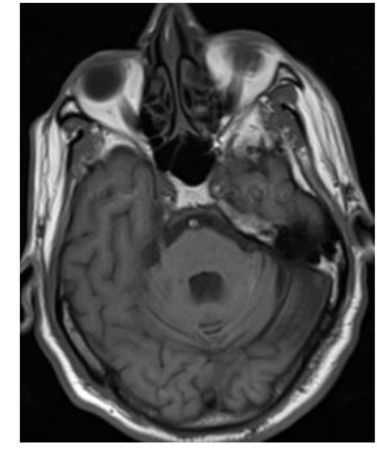

(a)

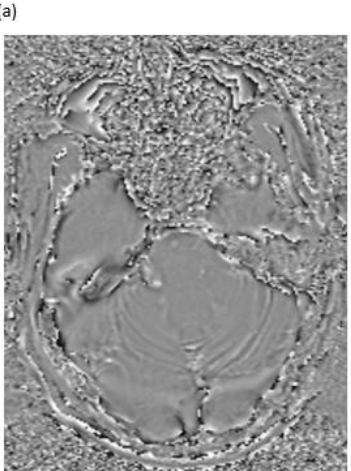

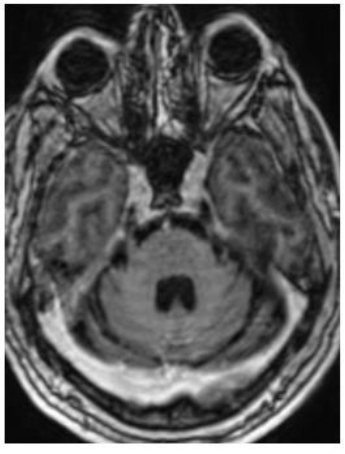

Figure 3. MRI in the region of the right trigeminal nerve does not demonstrate any significant abnormality on the conventional T1 WI (a) and post contrast T1 WI (b). SWI phase images (c) demonstrates an oblong hyperintensity in the region of the right trigeminal nerve suggestive of cavernous malformation.

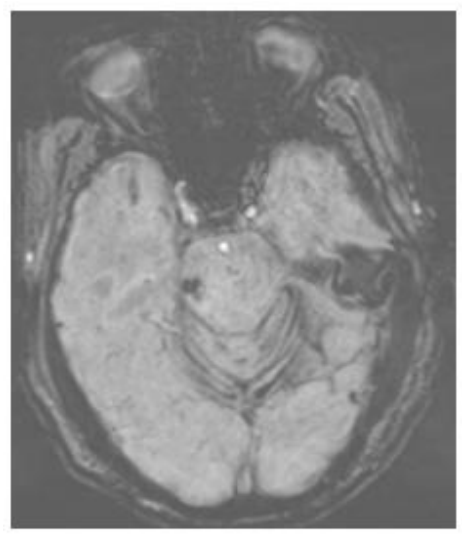

(a)

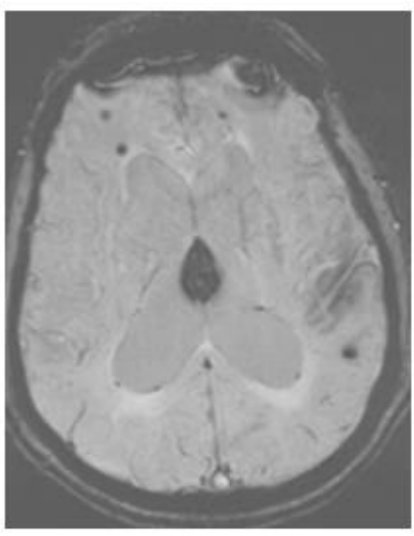

(b)

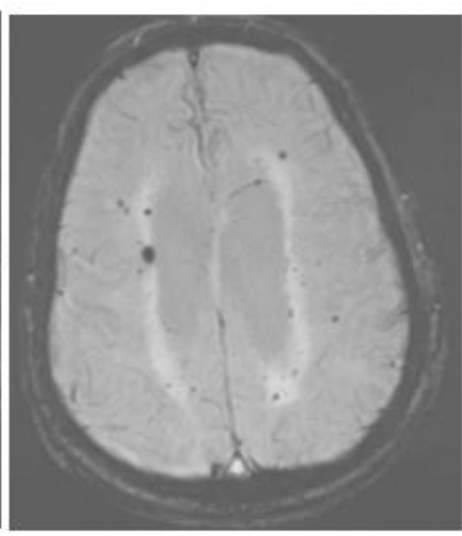

(c)

Figure 4. SW images demonstrating linear hypointensities along the cerebellar foliae (a), representing superficial siderosis, indicating that the trigeminal nerve cavernous malformation had bled previously. Multiple Tiny SWI hypointensities are seen distributed in bilateral cerebral hemispheres (b, c), suggesting microbleeds.

\section{Discussion}

Occurrence of cavernous malformations at sites other than cerebral parenchyma is rare. Only few case reports are available in the literature describing cavernous malformation in the septum pellucidum. Similarly, very few case reports are available describing cavernous malformations along the cranial nerves especially the trigeminal nerve.

The most common presenting symptom of cavernous malformations was headache. Other symptoms described were memory impairment and seizures. The other findings on 
imaging were intralesional bleeding at the time of presentation and hydrocephalus. Cavernous malformations may rupture and cause large hematomas, leading to neurological impairment. Bleeding is more common in infratentorial cavernous malformations.

Cavernous malformation can be familial or sporadic, or may appear following brain radiotherapy in rare cases.

A cavernous malformation within septum pellucidum may damage the bilateral fornices, which play an important role in memory. So, a septum pellucidum cavernous malformation should be surgically removed without further delay.

The neurosurgical approach should be such that damage to neighbouring vital structures is minimal, particularly those related to memory, like the fornices.

Mazumdar et.al. reported cavernous malformation of septum pellucidum at the level of foramen of monro causing obstructive hydrocephalus [1]. Patient presented with multiple episodes of severe headache and the lesion was resected by interhemispheric transcallosal route.

Narisawa et.al. also reported similar cases where patients' predominant symptom was headache [2].

Katoh et.al. reported cavernous malformation of septum pellucidum and in the left putamen. Patient presented with complaints of dementia as in our case [3].

Picolas et.al. also reported a case of cavernous malformation of septum pellucidum which was surgically resected. Patient presented with severe headache [4].

Pereira de Morais et.al. reported a case of trigeminal cavernous malformation causing trigeminal neuralgia [5]. Our patient didn't have any symptoms pertaining to trigeminal nerve.

Adachi et.al. reviewed cavernous malformations of trigeminal nerves presenting with trigeminal neuralgia [6]. Classification of cavernous malformations was done according to their origin, in the Gasserian ganglion (Type G), in the cisternal portion of the nerve (Type C), Intra-axial trigeminal nerve root in pons (Type $\mathrm{P}$ ) and in spinal tract of trigeminal nerve root (Type S) and treatment strategies were discussed according to the site of origin. In our case it was involving the cisternal portion of the nerve and intra-axial trigeminal nerve root in pons.

No surgical intervention was performed in our case.

\section{Conclusion}

To our knowledge, this is the first case report of simultaneous cavernous malformations involving the septum pellucidum and the trigeminal nerve.

Although there is no histopathology confirmation of the lesions, the imaging appearances are characteristic of cavernous malformations. Also, our case has superficial siderosis due to hemorrhage and hydrocephalus which was observed in other reported cases.

\section{References:}

1. Muzumdar D, Avinash KM, Ramdasi R. Cavernoma of the septum pellucidum in the region of foramen of Monro. Neurol India. 2015; 63(1):68-71. doi:10.4103/0028-3886.152641

2. Narisawa A, Kumabe T, Anzai T, Utsumi Y, Tominaga T. A case of cavernous angioma at the septum pellucidum. J. Neurological surgery. 2007; 35(1):53-58.

3. Katoh M, Sawamura Y, Moriwaki T, et al. A case of cavernous angioma in the septum pellucidum. Clin Neurol Neurosurg. 2013; 115(7):1126 - 1127. doi: 10.1016/j.clineuro.2012.09.010

4. Picolas C, Faropoulos K, Kekempanou K, Gatzounis G. Case Report of a Septum Pellucidum Cavernoma Surgically Resected via Inferior Parietal Approach and Short Literature Review. Open J Mod Neurosurg. Published online 2016. doi:10.4236/ojmn.2016.62013

5. Pereira de Morais NM, Mascarenhas AR, Soares-Fernandes JP, Moreira da Costa JA. Cranial nerve cavernous malformations causing trigeminal neuralgia and chiasmal apoplexy: Report of 2 cases and review of the literature. Surg Neurol Int 2012; 3:105.

6. Adachi K, Hasegawa M, Hayashi T, Nagahisa S, Hirose Y. A review of cavernous malformations with trigeminal neuralgia. Clin Neurol Neurosurg. 2014 Oct; 125:151-4. Doi: 10.1016/j.clineuro.2014.07.025. Epub 2014 Jul 30. PMID: 25129386. 